\title{
Reviewing the Role of Ultra-Widefield Imaging in Inherited Retinal Dystrophies
}

\author{
Maria Vittoria Cicinelli - Alessandro Marchese - Alessandro Bordato • \\ Maria Pia Manitto · Francesco Bandello · Maurizio Battaglia Parodi
}

Received: January 3, 2020 / Published online: March 5, 2020

(C) The Author(s) 2020

\section{ABSTRACT}

Inherited retinal dystrophies (IRD) are a heterogeneous group of rare chronic disorders caused by genetically determined degeneration of photoreceptors and retinal pigment epithelium cells. Ultra-widefield (UWF) imaging is a useful diagnostic tool for evaluating retinal integrity in IRD, including Stargardt disease, retinitis pigmentosa, cone dystrophies, and Best vitelliform dystrophy. Color or pseudocolor and fundus autofluorescence images obtained with UWF provide previously unavailable information on the retinal periphery, which correlates well with visual field measurement or electroretinogram. Despite unavoidable artifacts of the UWF device, the feasibility of investigations in infants and in patients with poor fixation makes UWF imaging a precious resource in the diagnostic armamentarium for IRD.

Keywords: Best vitelliform disease; Fundus autofluorescence; Inherited retinal dystrophy;

Enhanced digital features To view enhanced digital features for this article go to https://doi.org/10.6084/ m9.figshare.11882325.

M. V. Cicinelli $(\bowtie) \cdot A$. Marchese - A. Bordato M. P. Manitto · F. Bandello · M. Battaglia Parodi Department of Ophthalmology, San Raffaele VitaSalute University, Ospedale San Raffaele, Milan, Italy

e-mail: cicinelli.mariavittoria@hsr.it
Retinitis pigmentosa; Stargardt dystrophy; Ultra-widefield imaging

\section{Key Summary Points}

Inherited retinal dystrophies (IRD) are a heterogeneous group of rare chronic disorders caused by genetically determined degeneration of photoreceptors or retinal pigment epithelium (RPE) cells.

Color and fundus autofluorescence ultrawidefield (UWF) imaging have added novel insights in the interpretation of both macular and peripheral changes occurring in IRD.

UWF imaging aids in the diagnosis and monitoring of patients with IRD; UWF changes correlate well with functional damage on visual field test and electroretinogram.

Advantages of digital UWF imaging systems include enhanced resolution, easier acquisition with non-compliant patients (i.e., children), and avoidance of pupil dilation.

Future investigations on structure-function and phenotype-genotype correlations using UWF will increase our understanding of the complex spectrum of IRD. 


\section{INTRODUCTION}

Inherited retinal dystrophies (IRD) are a heterogeneous group of rare chronic disorders caused by genetically determined degeneration of photoreceptors or retinal pigment epithelium (RPE) cells. IRD are heterogeneous in terms of onset of symptoms and severity of clinical manifestations. IRD can be clinically categorized into four groups: rod-dominant diseases, cone-dominant diseases, generalized retinal degenerations, and vitreoretinal disorders $[1,2]$. An additional group of IRD includes pure macular diseases, namely Stargardt disease (STGD1), pattern dystrophy, Best vitelliform dystrophy (BVD), Sorsby disease, North Carolina dystrophy, and Doyne honeycomb dystrophy [3].

Specific complaints, age of onset, inheritance pattern, and electro-functional tests (such as full-field electroretinogram (ERG) and multifocal ERG) may suggest the site of retinal dysfunction and, in specific cases, the gene defect.

There is currently no specific treatment for any form of retinal dystrophy; antioxidant drugs, such as lutein, photoprotection, and lowvision aids are the only therapeutic alternatives offered nowadays. Nevertheless, early diagnosis and better characterization are important for accurate information on prognosis, genetic counseling, and gene-targeted therapeutic options available in the future. The aim of the present narrative review is to elucidate the role of non-invasive imaging in IRD, focusing on widefield (WF) and ultra-widefield (UWF) imaging devices. This article is based on previously conducted studies and does not contain any studies with human participants or animals performed by any of the authors.

\section{NON-INVASIVE IMAGING AND WIDEFIELD APPLICATION IN IRD}

Fundus autofluorescence (FAF) is one of the most informative techniques in patients with IRD. Two types of FAF are mostly used in clinical practice: short wavelength autofluorescence (SW-FAF), with an emitting light of $488 \mathrm{~nm}$, is specific for lipofuscin, and near-infrared autofluorescence (NIR-FAF), with an emitting light of $787 \mathrm{~nm}$, is specific for melanin. The changes on SW- and NIR-FAF provide useful information about the pathophysiology of IRD. SW-FAF shows hyper-autofluorescence in the case of lipofuscin buildup [4] or outer retinal disruption [5]; on the other hand, hypo-autofluorescence corresponds to areas of RPE loss (as RPE atrophy) or masquerading of normal RPE autofluorescence (such as pigment clumping) [6-8]. SW-FAF signal may also transiently increase after blue light exposure; this phenomenon has been called photobleaching. The optical pigments of photoreceptors normally absorb light in the same spectrum of SW-FAF; illumination with blue light induces an isomerization of the optical pigments from the 11-cis to all-trans conformation, resulting in a temporary loss of their absorption properties [9]. Photobleaching might differ between healthy and diseased eyes, including intermediate age-related macular degeneration [10] and IRD [11].

Fundus fluorescein angiography (FFA) and indocyanine green angiography (ICGA) evaluate the retinal and choroidal perfusion [12] and the presence of rare complications of IRD, such as cystoid macular edema (CME) or choroidal neovascularization (CNV) [13]. While they are useful at the first diagnosis, they are poorly applicable in monitoring patients with IRD.

Optical coherence tomography (OCT) assesses the state of the vitreoretinal interface, the inner retinal layers, the photoreceptors, and the RPE; OCT also confirms the presence of specific features, including vitelliform accumulation in Best disease, flecks in STGD1, and foveal cavitation in certain cone-rod dystrophies $[14,15]$. OCT angiography (OCTA) has added novel insights in the interpretation of vascular changes occurring in the macula in patients with IRDs. OCTA combines information from morphology and perfusion at the level of the superficial capillary plexus and the deep capillary plexus of the inner retina, at the choriocapillaris, and the choroidal layer. OCTA has shown interesting changes in a wide variety of IRD, including STGD1 [16], retinitis pigmentosa (RP) [17], and BVD [18]. Whereas the scanning 
range of most commercially available OCTA devices is still limited to the posterior pole, technical advances now allow scanning of larger areas of the retina (up to $80^{\circ}$ ) by montage of multiple scans $[19,20]$. A swept-source OCTA device (PlexElite, Carl Zeiss Meditec, Dublin, CA, USA) has been applied to investigate the grade of peripheral perfusion impairment in patients with RP [21]; further studies are needed to clarify the peripheral vascular involvement in other IRDs.

Traditional fundus retinography and fluorangiographic photograms include only the central $30^{\circ}$ or $55^{\circ}$ of the retina; this field of view is optimal for detecting pathologic changes of the optic nerve head, the papillary region, and the macula, but is inadequate for the imaging of the medium-far and of the very-far retinal periphery. In recent years, dedicated non-mydriatic wide-angle digital cameras have been developed to obtain UWF imaging of the retina, including the Optos Optomap ${ }^{\circledR}$ (Dumfermline, Scotland, UK), the ZEISS Clarus 500 (Dublin, USA) [22], and the Centervue Eidon device (Padova, Italy) [23]. UWF imaging allows simultaneous evaluation of the peripheral and central retina without the requirement of eye steering; UWF imaging has revealed interesting details about peripheral retinal lesions in IRDs $[24,25]$.

Advantages of digital UWF imaging systems include enhanced resolution, short image acquisition and processing time, the possibility of image manipulation and transmission, goodquality capturing in eyes with cataract or media opacities, easier procedure with non-compliant pediatric patients, and avoidance of pupil dilation $[26,27]$. UWF systems combine standard fundus photography (either true color or pseudocolor) with other retinal investigations, namely FFA, ICGA, red-free filter, and FAF. While confocal scanning laser ophthalmoscopy (cSLO) such as the Heidelberg Spectralis operates with SW-FAF and NIR-FAF filters only, UWF devices are coupled with novel technologies for FAF signal generation. Optos Optomap is embedded with a green-light autofluorescence (excitation $518 \mathrm{~nm}$ ); as green-light autofluorescence has a smaller rate of absorption by macular pigments, it better discriminates FAF anomalies in the perifoveal retina [28]. Eidon contains a "true color" confocal scanner which captures images with three different sources: LED (440-650 nm), infrared (825-870 nm), and blue light (440-475 $\mathrm{nm})$. The Eidon system separates short and long FAF emission wavelengths, obtaining a spectrally resolved FAF or color-FAF [29-31]. This imaging technique has revealed interesting new details in patients with IRD [32].

\section{STARGARDT DISEASE}

STGD1 is a recessive inherited disease with an incidence of $8-10$ per 100,000 persons, caused by autosomal mutations in the $A B C A 4$, ELOVL4, or PROM1 genes [33, 34]. The pathognomonic features of STGD1 include yellow or white fish-shaped flecks and photoreceptors, RPE, and choriocapillaris atrophy at the posterior pole. STGD1 is a progressive disease; however, the rate of disease progression changes varies considerably between subjects [35]. The number of flecks tends to increase with time with a radial, centrifugal pattern; indeed, UWF imaging is able to show lesions extending beyond the vascular arcades, in contrast to conventional imaging (Fig. 1). Both SW-FAF and NIR-FAF are reliable techniques for monitoring the progression of the disease [36, 37]. Moreover, spectrally resolved FAF allowed the distinction of two types of flecks: the first type is located centrally and shows green emission fluorescent component $(500-560 \mathrm{~nm})$ and disruption of outer retinal bands on OCT; the other type is located mainly peripherally and shows red emission fluorescent component $(560-700 \mathrm{~nm})$ and only minor alterations of outer retinal layers on OCT [32]. RPE atrophy can also spread outside the vascular arcades in later stages of STGD1 disease, sparing the peripapillary region (Fig. 2) [38, 39].

A novel classification of UWF FAF patterns in STGD1 has been published and is based on the distribution of flecks and RPE atrophy extension (Table 1) [40]. UWF imaging is particularly useful in characterizing the type III pattern, in which RPE atrophy extends towards the equator. The type III pattern has the worst prognosis: 


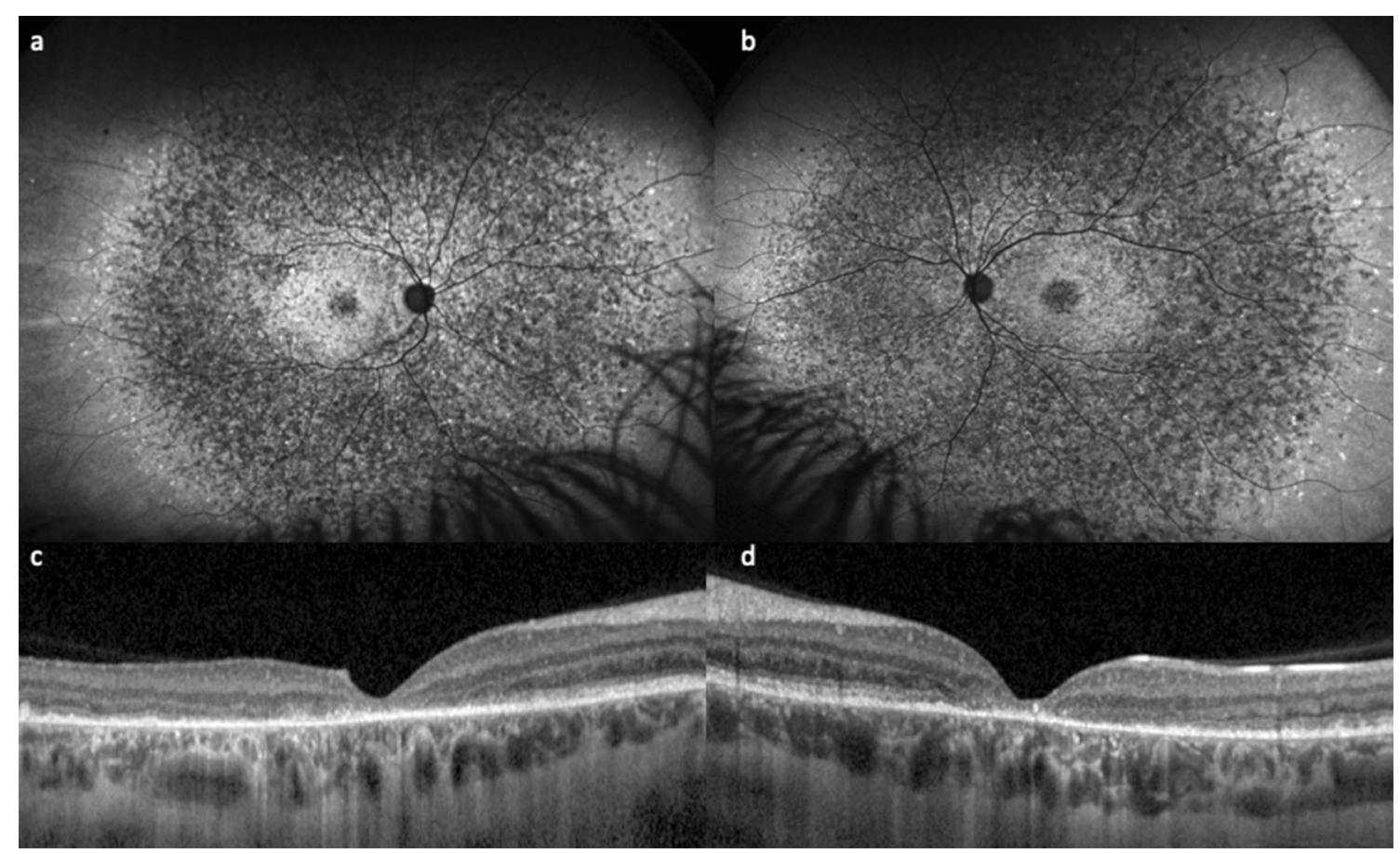

Fig. 1 Ultra-widefield fundus autofluorescence of a patient with Stargardt disease. a, b Diffuse, symmetrical hyper- and hypo-autofluorescent lesions involve the posterior pole and extend far beyond the vascular arcades. Conventional imaging might miss the peripheral flecks.

it is associated with severe alterations on OCT and vascular capillary damage in the central retina on OCTA, compared with other patterns [41]. Moreover, patients with widespread disease on UWF imaging present with a greater constriction of peripheral isopters, more dysfunction on ERG, and worse best corrected visual acuity (BCVA) compared to patients with disease confined to the macula [42].

Less frequent findings have been described on UWF FAF, including a hyper-autofluorescent ring at the transition between diseased and healthy retina, an oval-shaped hyper-autofluorescence outside the arcades, and mottled peripheral FAF changes (Fig. 3) [43]. Recently, peripheral pigmented retinal lesions resembling congenital hypertrophy of the RPE (CHRPE) have also been reported in patients with STGD1 $[44,45]$. Patients with peripheral pigmented hypertrophy featured decreased visual acuity, large central scotomas, abnormal ERG, and more widespread involvement of the peripheral
Moreover, UWF imaging allows for objective disease classification (see text for details). c, d Optical coherence tomography of the macular region, disclosing atrophy of the outer retinal layers subfoveally

retina compared with patients without CHRPElike lesions.

In conclusion, UWF imaging has allowed a shifting paradigm in STGD1 and other ABCA4related diseases from a pure macular dystrophy to a more generalized affection. Although STGD1 predominantly involves the macula in the first stages, significant retinal changes extend to the periphery with the progression of the disease. According to some authors, type III STGD1 with peripheral involvement represents a different phenotype of STGD1 rather than a progression of center-involving disease.

\section{RETINITIS PIGMENTOSA AND PIGMENTARY DYSTROPHIES}

The term retinitis pigmentosa (RP) embraces a heterogeneous group of inherited dystrophies characterized by progressive degeneration of rods and secondary involvement of cones. RP 


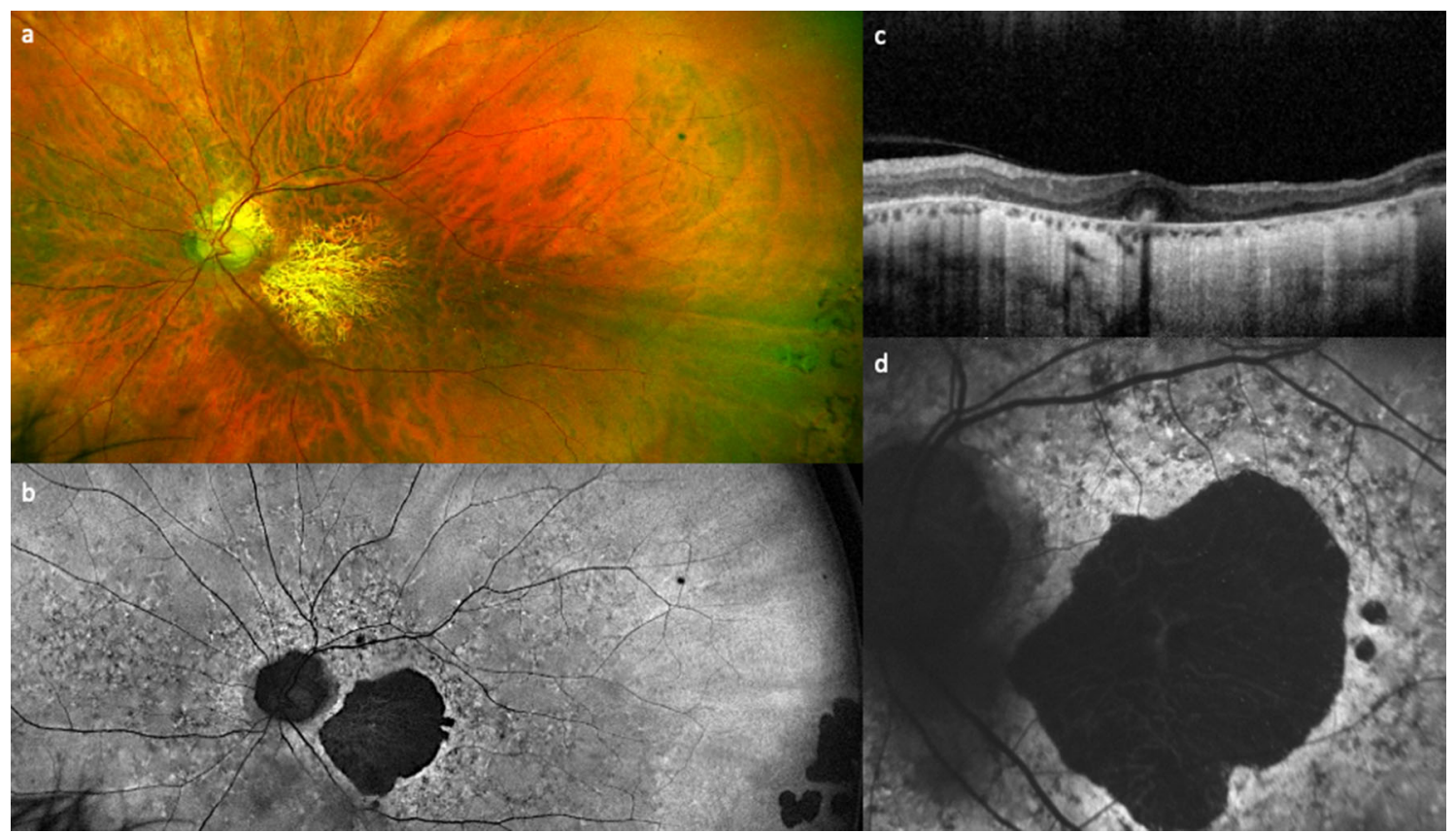

Fig. 2 Peripapillary sparing on fundus autofluorescence in a patient with Stargardt disease. a, b Ultra-widefield pseudocolor and green-light fundus autofluorescence. c Optical coherence tomography of the macula, disclosing extensive atrophy of the photoreceptors and the retinal pigment epithelium (RPE). d Short-wavelength fundus autofluorescence performed the year before, showing a smaller area of RPE atrophy at the posterior pole

Table 1 Ultra-widefield classification of Stargardt disease (STGD1) based on ultra-widefield fundus autofluorescence (Adapted from Klufas et al. [40])

\begin{tabular}{ll}
\hline STGD1 phenotype & Description \\
\hline Type I & Central atrophy \\
& Flecks confined to posterior pole \\
& No alterations outside $55^{\circ}$ field of view \\
& Central atrophy \\
Type II & Flecks extending outside posterior pole \\
& Central atrophy \\
Type III & Extramacular flecks \\
& Atrophy extending outside posterior pole \\
Type IIIA & Mild/moderate atrophy (punctate pattern) extending to or beyond equator \\
Type IIIB & Severe atrophy extending from macula to equator \\
Type IIIC & Extensive atrophy extending anterior to equator \\
\hline
\end{tabular}

can be inherited as autosomal dominant, autosomal recessive, or X-linked disease; rarely, it can originate from spontaneous mutations [46].
Night blindness and peripheral visual field defect are the most common symptoms at the onset of RP; gradual central visual acuity loss 


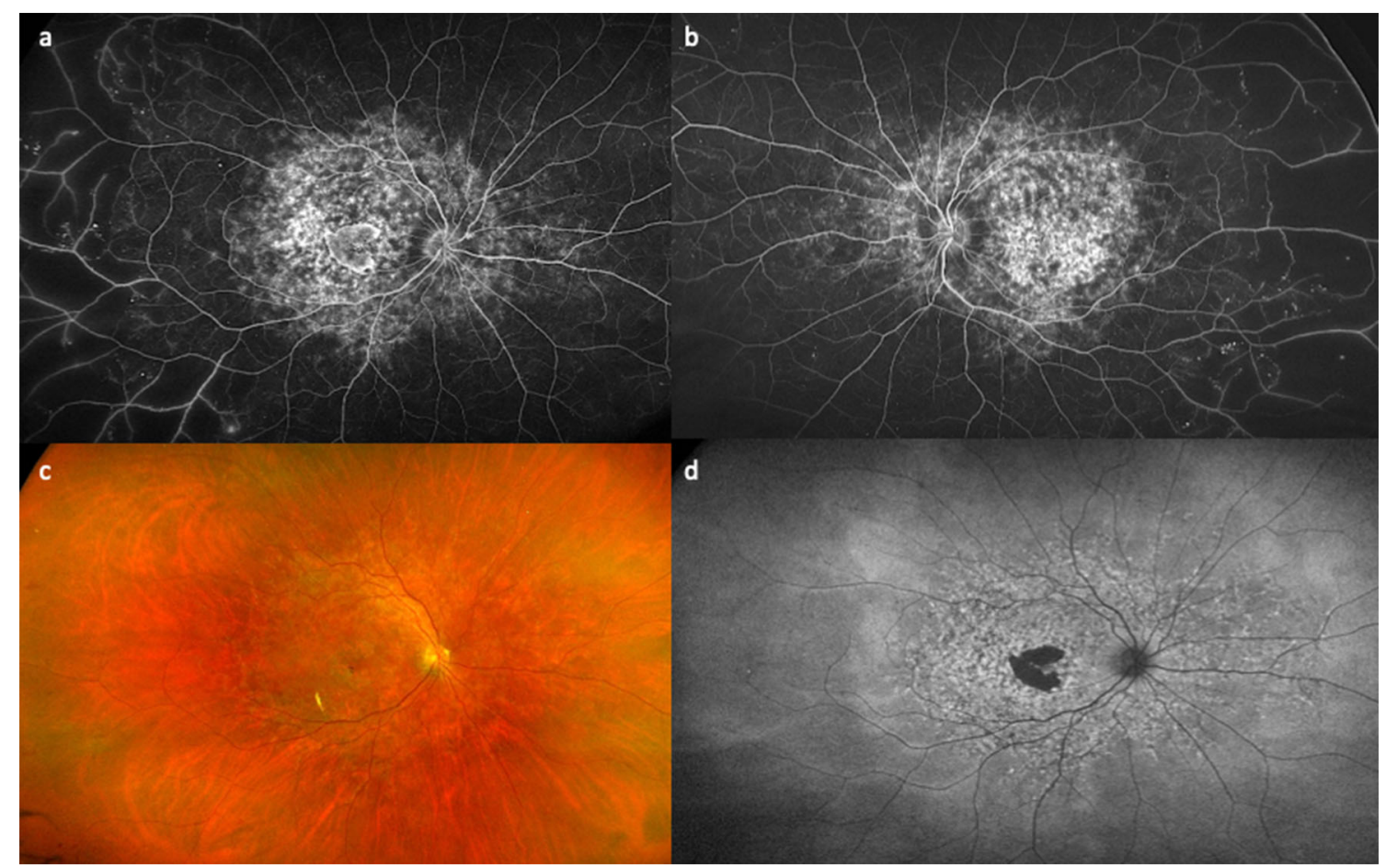

Fig. 3 Peripheral changes on ultra-widefield imaging in a patient with Stargardt disease and concurrent diabetic retinopathy. a, b Fundus fluorescein angiography showing extensive window effect at the posterior pole and coalescent areas of retinal non-perfusion in the periphery. c,

may take place in advanced stages. The pathogenesis of RP is complex: loss of photoreceptors is accompanied by changes in the RPE and retinal glia; ultimately, the inner retinal neurons, blood vessels, and the optic nerve head are affected. Pigment clumping in the mid and extreme periphery, vessel attenuation, and CME are the typical fundus findings.

UWF imaging is revealed a precious tool to diagnose and monitor patients suffering from $\mathrm{RP}$; a complex grading of RP changes has been recently proposed that is based on UWF FAF (Table 2) [25]. Different phenotypes of decreased autofluorescence (DAF) have been described in RP; patients with widespread DAF (grade 4) (especially if featuring nummular DAF) are significantly older than other groups (Fig. 4) [25].

Patients with RP exhibit a ring of perifoveal hyper-autofluorescence; this line is thought to be the landmark between preserved central d Pseudocolor and green-light fundus autofluorescence disclose macular hypo-autofluorescence due to retinal pigment epithelium atrophy. In the temporal periphery, a sharp shift from normal fluorescence to diffuse hypoautofluorescence is noticeable (only right eye shown)

fovea and diseased retina [47-49]. The area external to this ring correlates with the extent of visual field damage [50-52]. UWF FAF has a greater predictive value for estimating residual retinal sensitivity than OCT [52]; in fact, hypoautofluorescence on UWF FAF correlates with functional scotomas on Goldmann perimetry with an agreement of $91 \%$, whereas normal autofluorescence corresponds to normal visual fields with an agreement of 85\% [53].

In selected cases, UWF imaging also allows genotypic/phenotypic correlation in patients with RP. Patients with RHO, USH2A, CEP290, $R P G R I P 1$, and RPGR mutations usually show a prominent hyper-autofluorescent ring at the macula; patients with PRPF31 mutations show a similar ring of autofluorescence in the macula and a circular pattern of coarse DAF in the midperiphery [25]. A second ring of macular hyperautofluorescence has been found in patients with USH2A mutations. A peripheral patchy 
Table 2 Ultra-widefield classification of retinitis pigmentosa (RP) based on ultra-widefield fundus autofluorescence (Adapted from Hariri et al. [25])

\begin{tabular}{l}
\hline RP Description \\
changes
\end{tabular}

Macular abnormal autofluorescence

Grade 0 Neither ring nor central abnormal autofluorescence

Grade 1 Abnormal ring autofluorescence

Grade 2 Abnormal central autofluorescence

Presence of radial hyper-autofluorescence

Present

Absent

Extension of DAF

Grade 0 No DAF

Grade 1 Far peripheral DAF (outside of 7 standard ETDRS fields)

Grade 2 Central DAF (ETDRS fields 1, 2, and 3 involved)

Grade Only 1 field shows DAF

$2 \mathrm{~A}$

Grade 2 fields show DAF

2B

Grade All 3 fields show DAF

$2 \mathrm{C}$

Grade 3 Central and peripheral DAF (ETDRS fields 1, 2 , and 3 involved and more than $25 \%$ of the retina outside of the 3 central ETDRS fields)

Grade 4 Widespread DAF

Type 1 Fine granular DAF

Type 2 Branching DAF

Type 3 Coarse DAF

Type 4 Nummular DAF

Disc hyper-autofluorescence

Grade 0 No disc hyper-autofluorescence

Grade 1 Partial disc hyper-autofluorescence
Table 2 continued

RP Description
changes

Grade 2 Complete disc hyper-autofluorescence

$D A F$ decreased autofluorescence, ETDRS Early Treatment Diabetic Retinopathy Study

pattern of hyper/hypo-autofluorescence has been described in patients with $R H O$ or $R P G R$ mutations, while diffuse hypo-autofluorescence is distinctive for USH2A or RDS/PRPH2 mutations [54]. Patients with X-linked RP present radial hyper-autofluorescence starting from the mid-periphery and pointing towards the posterior pole $[55,56]$. Finally, one patient with CRB1 mutation demonstrated para-arterial preservation of the RPE [25].

Pigmented paravenous retinochoroidal atrophy (PPRCA) is a rare retinal dystrophy characterized by bilateral retinal and RPE atrophy and pigment clumping along the large retinal veins; PPRCA is part of the spectrum of RP $[57,58]$. The diagnosis is clinical and its exact pathogenesis is not clear. On UWF pseudocolor photographs, pigment appears as bone spicule, coarse clumps, or fine granular accumulations starting from the optic disc and expanding into the mid-periphery [59]. UWF FAF shows welldemarcated perivenular hypo-autofluorescence corresponding to RPE atrophy and pigmentation (Fig. 5) [59]. A crescent-like hyper-autofluorescent demarcation can be noticed at the border between the normal and affected retina or along the retinal veins, with or without central hypo-autofluorescence (Fig. 5) [60]. Widefield OCTA revealed a relative sparing of the retinal capillary plexuses (superficial and deep), with diffuse defects in the choriocapillaris corresponding to RPE atrophy [61].

\section{CONE DYSTROPHIES}

Cone dystrophies (CDs) and cone-rod dystrophies (CRDs) are the most common hereditary cone disorders [62]; CRDs differ from CDs in terms of the impairment of rod function from 


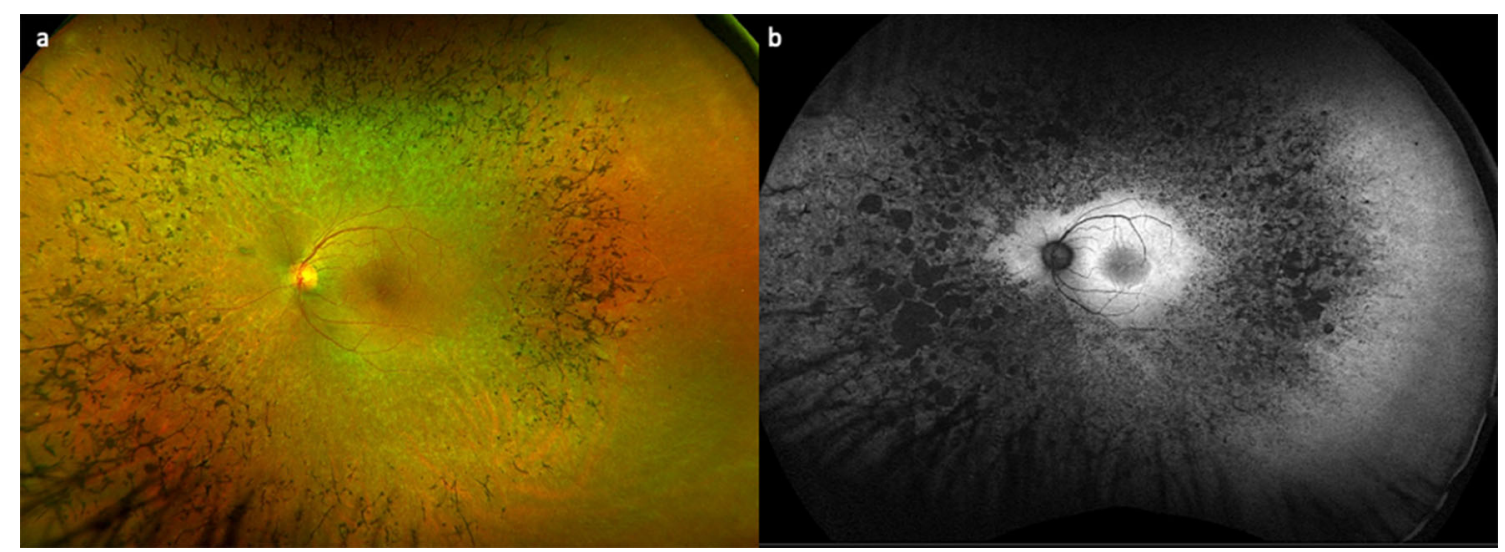

Fig. 4 Ultra-widefield imaging of a patient with retinitis pigmentosa. a Pseudocolor imaging revealing bone spiculae pigmentation in medium periphery and vascular attenuation. b Green-light fundus autofluorescence allows disease classification on the basis of the distribution of decreased autofluorescence (DAF) lesions; the patient presents widespread nummular DAF. Autofluorescence also shows abnormal central hypo-autofluorescence and a ring of hyper-autofluorescence in the perifoveal area

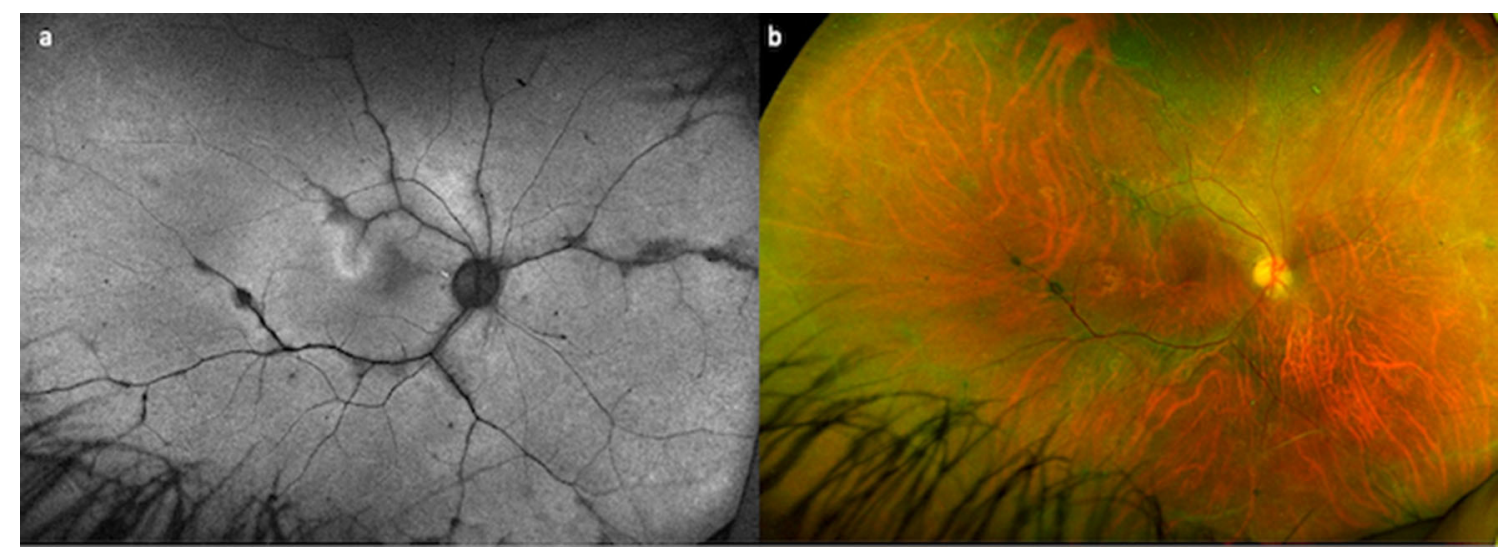

Fig. 5 Ultra-widefield imaging of a patient with perivenular pigmentary retinopathy. a Green-light fundus autofluorescence shows perivenular areas of hypo-autofluorescence corresponding to retinal pigment epithelium atrophy and

the early phases of the disease. Two additional forms of localized cone dysfunction syndromes have been described: occult macular dystrophy and peripheral cone dystrophy (PCD) [63, 64].

Fundus examination is normal in the majority of patients; in advanced cases, it may show a macular bullseye aspect with various pigmentation spreading towards the periphery. A crescentlike hyper-autofluorescent demarcation can be noted along the peripheral retinal veins. b Pseudocolor showing pigmentary changes along the main retinal vessels

degrees of retinal and RPE atrophy [65]. Patients with primary cone degeneration and secondary rod disruption may also develop retinal vascular attenuation and peripheral pigment deposits, mimicking RP. FAF, OCT, and OCTA macular abnormalities have been extensively described in patients with $\mathrm{CD}, \mathrm{CRD}$, and localized cone 


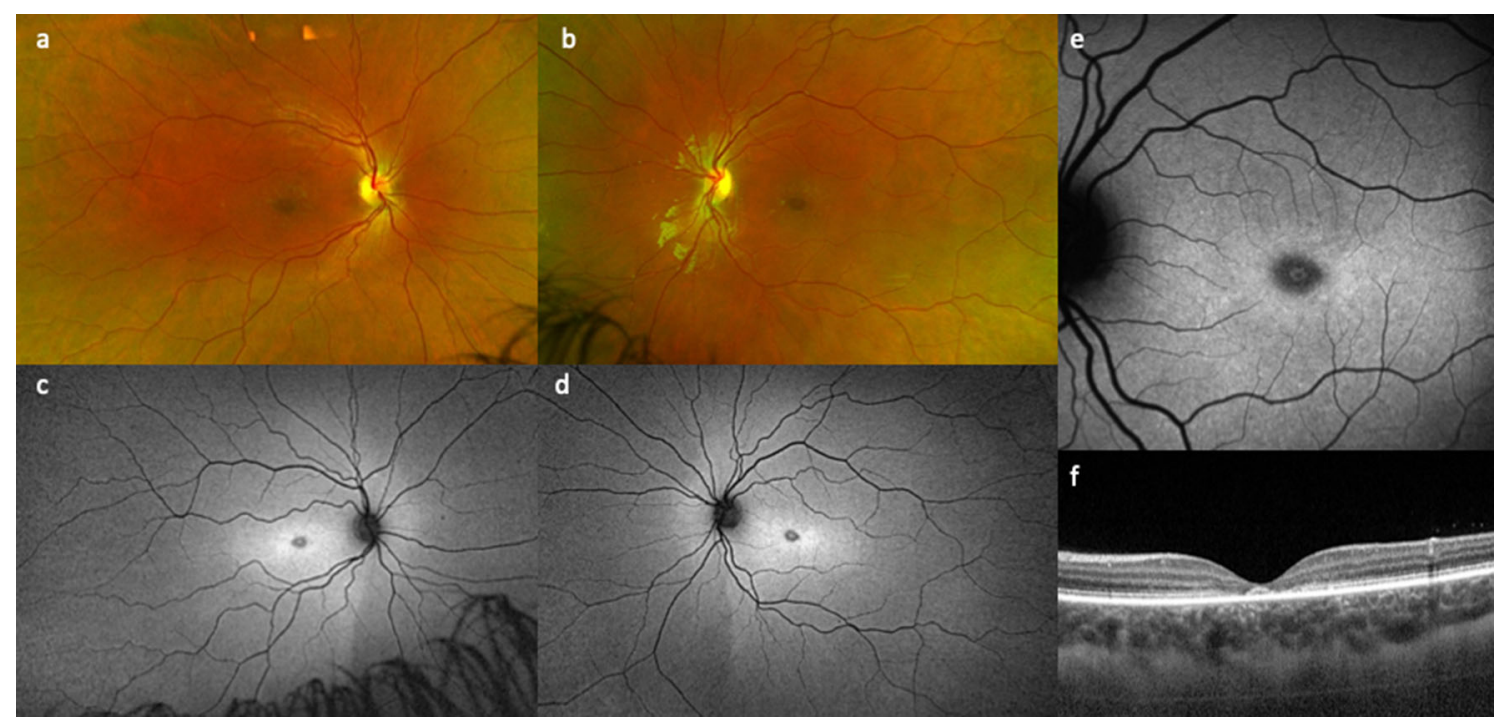

Fig. 6 Ultra-widefield imaging of a patient with cone dystrophy. a, $\mathbf{b}$ Alteration of the macular reflex on pseudocolor imaging. c, d Green-light fundus autofluorescence shows macular hypo-autofluorescence surrounded by

dysfunction syndromes [66-69]. On the other hand, reports on UWF changes in these patients are still scarce.

UWF FAF displays a hyper-autofluorescent macular ring in certain cases of CD and CRD (Fig. 6) [50]; in the periphery, abnormal FAF inversely correlates with the residual visual function on both Goldmann perimetry and ERG responses [70]. In a large intra-familial study, UWF FAF showed high similarity between siblings but significant diversity between parent-child pairs; this finding suggests that UWF imaging features in CD and CRD—or more generally with IRD—tend to change with age [71].

In patients with PCD, UWF FAF showed a central bullseye lesion and perimacular hyperautofluorescence with rounded or scalloped borders and, in the periphery, irregular hyper- a ring of hyper-autofluorescence. e A similar pattern is noticeable on short wavelength autofluorescence (only left eye shown). f Optical coherence tomography of the macula disclosing atrophy of the foveal cones

and hypo-autofluorescence located mainly in the nasal quadrant and not contiguous with the macular findings [72]. UWF imaging also aided in the differential diagnosis between PCD (which remains a diagnosis of exclusion) and other posterior segment conditions, such as retinal white dot syndromes, autoimmune retinopathies, infectious chorioretinitis, enhanced $\mathrm{S}$-cone syndrome, pericentral retinitis pigmentosa, or STGD1 [73].

\section{BESTROPHINOPATHIES}

Best vitelliform dystrophy (BVD) is an autosomal dominant macular dystrophy caused by the somatic mutation of the BEST1 gene; the disease belongs to a bigger family of conditions known as bestrophinopathies characterized by the 


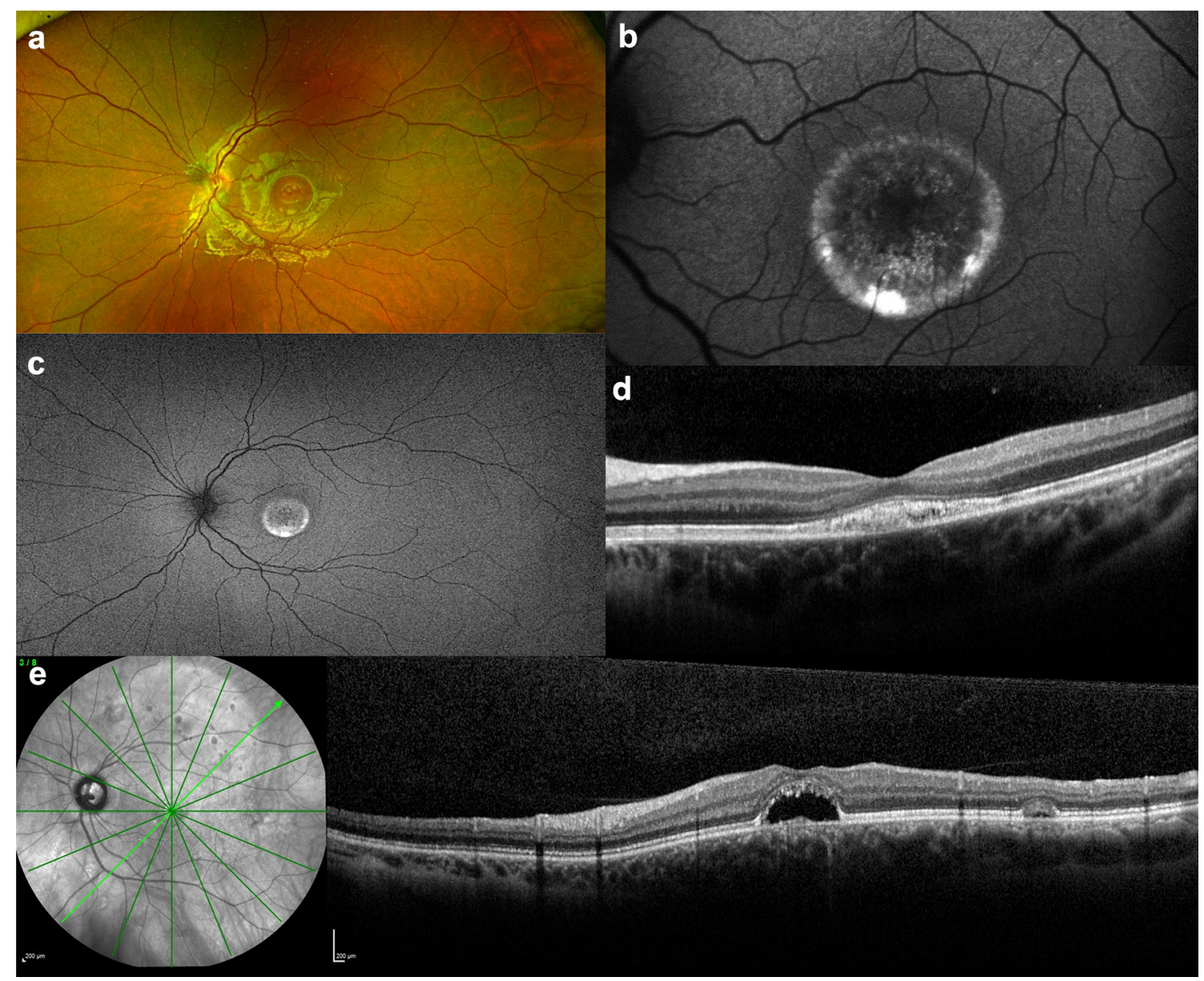

Fig. 7 Multimodal imaging of Best vitelliform dystrophy. a Pseudocolor showing a macular vitelliform lesion in a vitelliruptive stage. b, c Short wavelength and green autofluorescence showing dishomogeneous hyperfluorescence at the macular region. d Optical coherence

accumulation of lipofuscin and abnormal electro-oculographic (EOG) Arden ratio [74]. Although BVD usually presents with a single lesion (Fig. 7), up to $30 \%$ of patients feature multifocal lesions within and outside the foveal region (Fig. 8). In some patients, other lessspecific retinal lesions may be found, such as schisis, subretinal fibrosis, or pigment clumping tomography confirming the presence of vitelliform subfoveal material. e Optical coherence tomography of another case of Best vitelliform dystrophy, showing subretinal hyporeflective lesions (likely to be previous vitelliform material) both in the fovea and in the mid-periphery

[75]. Extrafoveal lesions are usually smaller than those located into the macula [76, 77]. Despite no study having systematically addressed the changes in the retinal periphery in BVD and other BEST1-related diseases, UWF imaging may significantly help in the assessment and the monitoring of the vitelliform lesions, especially those located in extrafoveal regions. 


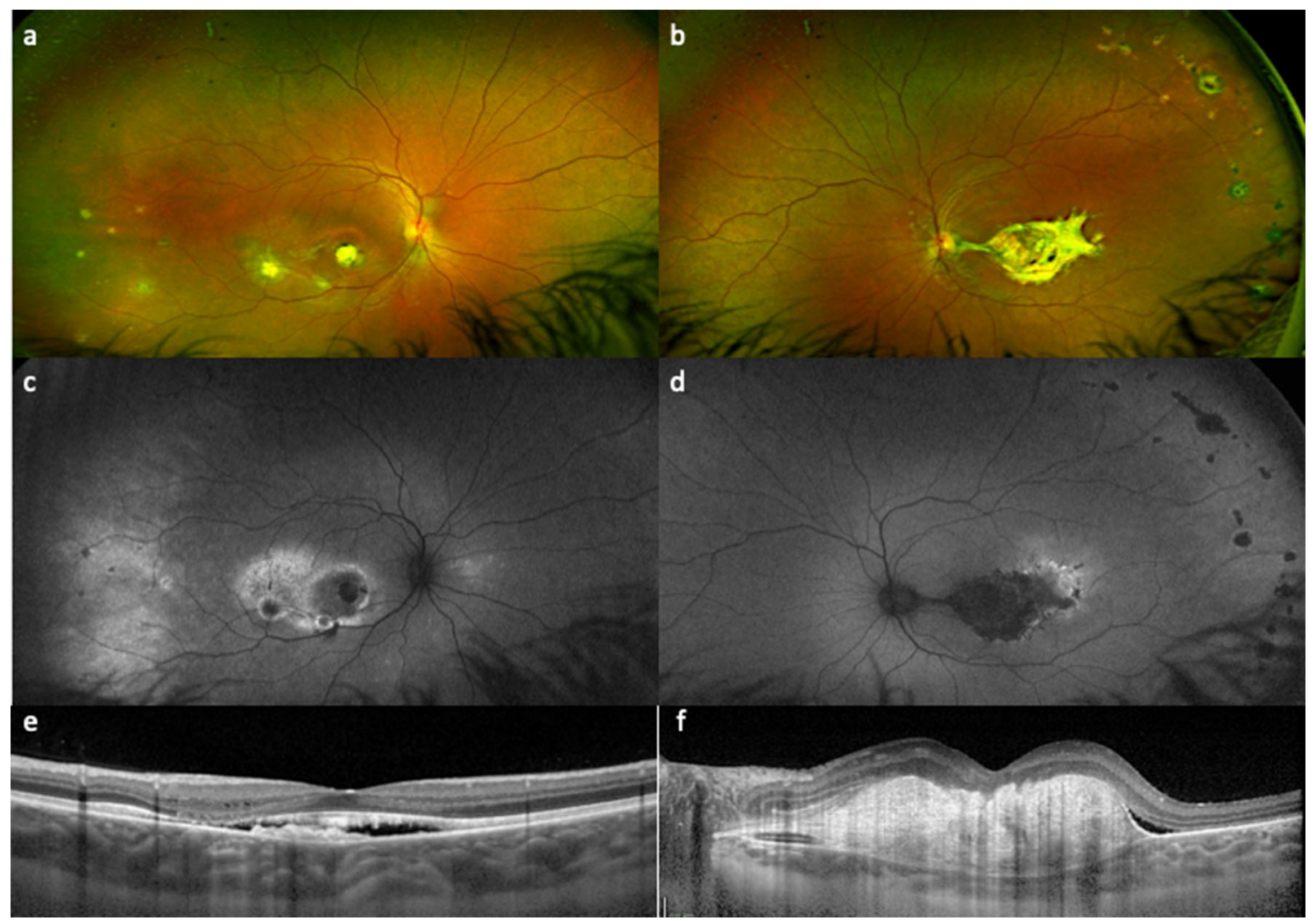

Fig. 8 Multimodal imaging of a patient with multifocal Best vitelliform dystrophy. a, b Pseudocolor showing multiple vitelliform lesion complicated by subretinal fibrosis both at the posterior pole and in the far periphery. c, d Green-light autofluorescence discloses mottled signal

\section{CONCLUSION}

Ultra-widefield imaging is a useful tool for evaluating retinal integrity in IRD. Color or pseudocolor and FAF images obtained with UWF provide previously unavailable information about the retinal periphery, which correlates well with visual field or ERG measurement in patients with IRD. Physicians should be aware of peripheral image magnification, image distortion, and artifacts of the UWF devices; nevertheless, the feasibility of investigations in infants and in patients with poor fixation makes UWF imaging a precious resource in the diagnostic armamentarium. Future investigations on structure-function and phenotype-genotype correlations using UWF imaging will undoubtedly increase our understanding of the complex spectrum of IRD. at the posterior pole and hypo-autofluorescence corresponding to the peripheral lesions. e, f Optical coherence tomography shows an almost completely reabsorbed vitelliform material in the right eye and extensive fibrosis in the left macula

\section{ACKNOWLEDGEMENTS}

Funding. No funding or sponsorship was received for this study or publication of this article.

Authorship. All named authors meet the International Committee of Medical Journal Editors (ICMJE) criteria for authorship for this article, take responsibility for the integrity of the work as a whole, and have given their approval for this version to be published.

Disclosures. Maria Vittoria Cicinelli, Alessandro Marchese, Maria Pia Manitto, Alessandro Bordato, and Maurizio Battaglia Parodi have no financial disclosures. Francesco Bandello is advisory board member for Allergan, 
Bayer, Boehringer-Ingelheim, Hoffmann La Roche, Novartis, NTC Pharma, Sifi, Thrombogenics, Zeiss. Francesco Bandello receives personal fees by Sooft. No conflicting relationship exists for any author.

Compliance with Ethics Guidelines. This article is based on previously conducted studies and does not contain any studies with human participants or animals performed by any of the authors.

Data Availability. Data sharing is not applicable to this article as no datasets were generated or analyzed during the current study.

Open Access. This article is licensed under a Creative Commons Attribution-NonCommercial 4.0 International License, which permits any non-commercial use, sharing, adaptation, distribution and reproduction in any medium or format, as long as you give appropriate credit to the original author(s) and the source, provide a link to the Creative Commons licence, and indicate if changes were made. The images or other third party material in this article are included in the article's Creative Commons licence, unless indicated otherwise in a credit line to the material. If material is not included in the article's Creative Commons licence and your intended use is not permitted by statutory regulation or exceeds the permitted use, you will need to obtain permission directly from the copyright holder. To view a copy of this licence, visit http://creativecommons.org/licenses/bync/4.0/.

\section{REFERENCES}

1. Moore AT. Cone and cone-rod dystrophies. J Med Genet. 1992;29(5):289-90.

2. Michaelides M, Hardcastle AJ, Hunt DM, et al. Progressive cone and cone-rod dystrophies: phenotypes and underlying molecular genetic basis. Surv Ophthalmol. 2006;51(3):232-58.

3. Rahman N, Georgiou M, Khan KN, et al. Macular dystrophies: clinical and imaging features, molecular genetics and therapeutic options. $\mathrm{Br} \mathrm{J}$
Ophthalmol. 2019. https://doi.org/10.1136/ bjophthalmol-2019-315086.

4. Delori FC, Dorey CK, Staurenghi G, et al. In vivo fluorescence of the ocular fundus exhibits retinal pigment epithelium lipofuscin characteristics. Investig Ophthalmol Vis Sci. 1995;36(3):718-29.

5. Freund KB, Mrejen S, Jung J, et al. Increased fundus autofluorescence related to outer retinal disruption. JAMA Ophthalmol. 2013;131(12):1645-9.

6. Parodi $\mathrm{MB}$, Iacono $\mathrm{P}$, Campa $\mathrm{C}$, et al. Fundus autofluorescence patterns in Best vitelliform macular dystrophy. Am J Ophthalmol. 2014;158(5): 1086-92.

7. Parodi MB, Iacono P, Del Turco C, et al. Functional assessment of the fundus autofluorescence pattern in Best vitelliform macular dystrophy. Graefes Arch Clin Exp Ophthalmol. 2016;254(7):1297-302.

8. Parodi MB, Iacono $\mathrm{P}$, Triolo $\mathrm{G}$, et al. Morphofunctional correlation of fundus autofluorescence in Stargardt disease. Br J Ophthalmol. 2015;99(10): 1354-9.

9. Theelen T, Berendschot TT, Boon CJ, et al. Analysis of visual pigment by fundus autofluorescence. Exp Eye Res. 2008;86(2):296-304.

10. Borrelli E, Costanzo E, Parravano M, et al. Impact of bleaching on photoreceptors in different intermediate AMD phenotypes. Transl Vis Sci Technol. 2019;8(6):5.

11. Bosch-Presegue L, Ramon E, Toledo D, et al. Alterations in the photoactivation pathway of rhodopsin mutants associated with retinitis pigmentosa. FEBS J. 2011;278(9):1493-505.

12. Giani A, Pellegrini M, Carini E, et al. The dark atrophy with indocyanine green angiography in Stargardt disease. Investig Ophthalmol Vis Sci. 2012;53(7):3999-4004.

13. Stanga PE, Downes SM, Ahuja RM, et al. Comparison of optical coherence tomography and fluorescein angiography in assessing macular edema in retinal dystrophies: preliminary results. Int Ophthalmol. 2001;23(4-6):321-5.

14. Battaglia Parodi M, Iacono P, Romano F, et al. Spectral domain optical coherence tomography features in different stages of Best vitelliform macular dystrophy. Retina. 2018;38(5):1041-6.

15. Parodi MB, Cicinelli MV, Iacono $\mathrm{P}$, et al. Multimodal imaging of foveal cavitation in retinal dystrophies. Graefes Arch Clin Exp Ophthalmol. 2017;255(2):271-9. 
16. Battaglia Parodi M, Cicinelli MV, Rabiolo A, et al. Vascular abnormalities in patients with Stargardt disease assessed with optical coherence tomography angiography. Br J Ophthalmol. 2017;101(6): 780-5.

17. Battaglia Parodi M, Cicinelli MV, Rabiolo A, et al. Vessel density analysis in patients with retinitis pigmentosa by means of optical coherence tomography angiography. Br J Ophthalmol. 2017;101(4): 428-32.

18. Parodi MB, Romano F, Cicinelli MV, et al. Retinal vascular impairment in Best vitelliform macular dystrophy assessed by means of optical coherence tomography angiography. Am J Ophthalmol. 2018;187:61-70.

19. Eastline M, Munk MR, Wolf S, et al. Repeatability of wide-field optical coherence tomography angiography in normal retina. Transl Vis Sci Technol. 2019;8(3):6.

20. Pellegrini M, Cozzi M, Staurenghi G, et al. Comparison of wide field optical coherence tomography angiography with extended field imaging and fluorescein angiography in retinal vascular disorders. PLoS One. 2019;14(4):e0214892.

21. Mastropasqua R, D'Aloisio R, De Nicola C, et al. Widefield swept source OCTA in retinitis pigmentosa. Diagnostics (Basel). 2020;10(1):E50.

22. Hirano T, Imai A, Kasamatsu H, et al. Assessment of diabetic retinopathy using two ultra-wide-field fundus imaging systems, the Clarus(R) and Optos systems. BMC Ophthalmol. 2018;18(1):332.

23. Sarao V, Veritti D, Borrelli E, et al. A comparison between a white LED confocal imaging system and a conventional flash fundus camera using chromaticity analysis. BMC Ophthalmol. 2019;19(1): 231.

24. Trichonas G, Traboulsi EI, Ehlers JP. Ultra-widefield fundus autofluorescence patterns in retinitis pigmentosa and other retinal dystrophies. Ophthalmic Genet. 2017;38(1):98-100.

25. Hariri AH, Gui W, Datoo O'Keefe GA, et al. Ultrawidefield fundus autofluorescence imaging of patients with retinitis pigmentosa: a standardized grading system in different genotypes. Ophthalmol Retina. 2018;2(7):735-45.

26. Khurram Butt D, Gurbaxani A, Kozak I. Ultra-widefield fundus autofluorescence for the detection of inherited retinal disease in difficult-to-examine children. J Pediatr Ophthalmol Strabismus. 2019;56(6):383-7.
27. Kothari N, Pineles S, Sarraf D, et al. Clinic-based ultra-wide field retinal imaging in a pediatric population. Int J Retina Vitreous. 2019;5(Suppl 1):21.

28. Manivannan A, Plskova J, Farrow A, et al. Ultrawide-field fluorescein angiography of the ocular fundus. Am J Ophthalmol. 2005;140(3):525-7.

29. Pfau M, Goerdt L, Schmitz-Valckenberg S, et al. Green-light autofluorescence versus combined blue-light autofluorescence and near-infrared reflectance imaging in geographic atrophy secondary to age-related macular degeneration. Investig Ophthalmol Vis Sci. 2017;58(6): BIO121-30.

30. Borrelli E, Nittala MG, Abdelfattah NS, et al. Comparison of short-wavelength blue-light autofluorescence and conventional blue-light autofluorescence in geographic atrophy. Br J Ophthalmol. 2018. https://doi.org/10.1136/bjophthalmol-2018311849.

31. Borrelli E, Lei J, Balasubramanian S, et al. Green emission fluorophores in eyes with atrophic agerelated macular degeneration: a colour fundus autofluorescence pilot study. $\mathrm{Br} \mathrm{J}$ Ophthalmol. 2018;102(6):827-32.

32. Dysli C, Muller PL, Birtel J, et al. Spectrally resolved fundus autofluorescence in ABCA4-related retinopathy. Investig Ophthalmol Vis Sci. 2019;60(1):274-81.

33. Yi J, Li S, Jia X, et al. Evaluation of the ELOVL4, PRPH2 and ABCA4 genes in patients with Stargardt macular degeneration. Mol Med Rep. 2012;6(5): 1045-9.

34. Palejwala NV, Gale MJ, Clark RF, et al. Insights into autosomal dominant Stargardt-like macular dystrophy through multimodality diagnostic imaging. Retina. 2016;36(1):119-30.

35. Cukras CA, Wong WT, Caruso R, et al. Centrifugal expansion of fundus autofluorescence patterns in Stargardt disease over time. Arch Ophthalmol. 2012;130(2):171-9.

36. Cicinelli MV, Battista M, Starace V, et al. Monitoring and management of the patient with Stargardt disease. Clin Optom (Auckl). 2019;11:151-65.

37. Strauss RW, Ho A, Munoz B, et al. The natural history of the progression of atrophy secondary to Stargardt disease (ProgStar) studies: design and baseline characteristics: ProgStar Report No. 1. Ophthalmology. 2016;123(4):817-28.

38. Burke TR, Rhee DW, Smith RT, et al. Quantification of peripapillary sparing and macular involvement 
in Stargardt disease (STGD1). Investig Ophthalmol Vis Sci. 2011;52(11):8006-15.

39. Kumar V. Insights into autofluorescence patterns in Stargardt macular dystrophy using ultra-wide-field imaging. Graefes Arch Clin Exp Ophthalmol. 2017;255(10):1917-22.

40. Klufas MA, Tsui I, Sadda SR, et al. Ultrawidefield autofluoresence in Abca4 Stargardt disease. Retina. 2018;38(2):403-15.

41. Arrigo A, Grazioli A, Romano F, et al. Multimodal evaluation of central and peripheral alterations in Stargardt disease: a pilot study. Br J Ophthalmol. 2019. https://doi.org/10.1136/bjophthalmol-2019315148.

42. Abalem MF, Otte B, Andrews C, et al. Peripheral visual fields in ABCA4 Stargardt disease and correlation with disease extent on ultra-widefield fundus autofluorescence. Am J Ophthalmol. 2017;184: 181-8.

43. Chen L, Lee W, de Carvalho JRL Jr, et al. Multiplatform imaging in ABCA4-associated disease. Sci Rep. 2019;9(1):6436.

44. Zhao PY, Abalem MF, Nadelman D, et al. Peripheral pigmented retinal lesions in Stargardt disease. Am J Ophthalmol. 2018;188:104-10.

45. Abalem MF, Omari AA, Schlegel D, et al. Macular hyperpigmentary changes in ABCA4-Stargardt disease. Int J Retina Vitreous. 2019;5:9.

46. Kaplan J, Bonneau D, Frezal J, et al. Clinical and genetic heterogeneity in retinitis pigmentosa. Hum Genet. 1990;85(6):635-42.

47. Wakabayashi T, Sawa M, Gomi F, et al. Correlation of fundus autofluorescence with photoreceptor morphology and functional changes in eyes with retinitis pigmentosa. Acta Ophthalmol. 2010;88(5): e177-83.

48. Lenassi E, Troeger E, Wilke $\mathrm{R}$, et al. Correlation between macular morphology and sensitivity in patients with retinitis pigmentosa and hyperautofluorescent ring. Investig Ophthalmol Vis Sci. 2012;53(1):47-52.

49. Robson AG, El-Amir A, Bailey C, et al. Pattern ERG correlates of abnormal fundus autofluorescence in patients with retinitis pigmentosa and normal visual acuity. Investig Ophthalmol Vis Sci. 2003;44(8):3544-50.

50. Oishi A, Oishi M, Ogino K, et al. Wide-field fundus autofluorescence for retinitis pigmentosa and cone/cone-rod dystrophy. Adv Exp Med Biol. 2016;854: 307-13.
51. Oishi A, Ogino K, Makiyama Y, et al. Wide-field fundus autofluorescence imaging of retinitis pigmentosa. Ophthalmology. 2013;120(9):1827-34.

52. Lee J, Asano S, Inoue T, et al. Investigating the usefulness of fundus autofluorescence in retinitis pigmentosa. Ophthalmol Retina. 2018;2(10):1062-70.

53. Ogura S, Yasukawa T, Kato A, et al. Wide-field fundus autofluorescence imaging to evaluate retinal function in patients with retinitis pigmentosa. Am J Ophthalmol. 2014;158(5):1093-8.

54. Trichonas G, Traboulsi EI, Ehlers JP. Correlation of ultra-widefield fundus autofluorescence patterns with the underlying genotype in retinal dystrophies and retinitis pigmentosa. Ophthalmic Genet. 2017;38(4):320-4.

55. Wegscheider E, Preising MN, Lorenz B. Fundus autofluorescence in carriers of X-linked recessive retinitis pigmentosa associated with mutations in RPGR, and correlation with electrophysiological and psychophysical data. Graefes Arch Clin Exp Ophthalmol. 2004;242(6):501-11.

56. Ogino K, Oishi M, Oishi A, et al. Radial fundus autofluorescence in the periphery in patients with $\mathrm{X}$-linked retinitis pigmentosa. Clin Ophthalmol. 2015;9:1467-74.

57. Ratra D, Chandrasekharan DP, Aruldas P, et al. Concurrent retinitis pigmentosa and pigmented paravenous retinochoroidal atrophy phenotypes in the same patient. Indian J Ophthalmol. 2016;64(10):775-7.

58. McKay GJ, Clarke S, Davis JA, et al. Pigmented paravenous chorioretinal atrophy is associated with a mutation within the crumbs homolog 1 (CRB1) gene. Investig Ophthalmol Vis Sci. 2005;46(1): 322-8.

59. Takagi S, Hirami Y, Takahashi M, et al. Use of widefield fundus camera, fundus autofluorescence, and OCT in cases of pigmented paravenous retinochoroidal atrophy. Ophthalmol Retina. 2018;2(1):79-81.

60. Kumar V, Kumawat D, Tewari R, et al. Ultra-wide field imaging of pigmented para-venous retinochoroidal atrophy. Eur J Ophthalmol. 2019;29(4): 444-52.

61. Cicinelli MV, Giuffre C, Rabiolo A, et al. Optical coherence tomography angiography of pigmented paravenous retinochoroidal atrophy. Ophthalmic Surg Lasers Imaging Retina. 2018;49(5):381-3.

62. Tsang SH, Sharma T. Progressive cone dystrophy and cone-rod dystrophy (XL, AD, and AR). Adv Exp Med Biol. 2018;1085:53-60. 
63. Miyake Y, Horiguchi M, Tomita N, et al. Occult macular dystrophy. Am J Ophthalmol. 1996;122(5): 644-53.

64. Kondo M, Miyake Y, Kondo N, et al. Peripheral cone dystrophy: a variant of cone dystrophy with predominant dysfunction in the peripheral cone system. Ophthalmology. 2004;111(4):732-9.

65. Thiadens AA, Phan TM, Zekveld-Vroon RC, et al. Clinical course, genetic etiology, and visual outcome in cone and cone-rod dystrophy. Ophthalmology. 2012;119(4):819-26.

66. D'Esposito F, Cennamo G, de Crecchio G, et al. Multimodal imaging in autosomal dominant conerod dystrophy caused by novel CRX variant. Ophthalmic Res. 2018;60(3):169-75.

67. Zahlava J, Lestak J, Karel I. Optical coherence tomography in progressive cone dystrophy. Biomed Pap Med Fac Univ Palacky Olomouc Czech Repub. 2014;158(4):628-34.

68. Park SJ, Woo SJ, Park KH, et al. Morphologic photoreceptor abnormality in occult macular dystrophy on spectral-domain optical coherence tomography. Investig Ophthalmol Vis Sci. 2010;51(7):3673-9.

69. Fujinami K, Tsunoda K, Hanazono G, et al. Fundus autofluorescence in autosomal dominant occult macular dystrophy. Arch Ophthalmol. 2011;129(5): 597-602.

70. Oishi M, Oishi A, Ogino K, et al. Wide-field fundus autofluorescence abnormalities and visual function in patients with cone and cone-rod dystrophies. Investig Ophthalmol Vis Sci. 2014;55(6):3572-7.

71. Furutani Y, Ogino K, Oishi A, et al. Intra-familial similarity of wide-field fundus autofluorescence in inherited retinal dystrophy. Adv Exp Med Biol. 2016;854:299-305.

72. Sisk RA, Hufnagel RB, Laham A, et al. Peripheral cone dystrophy: expanded clinical spectrum, multimodal and ultrawide-field imaging, and genomic analysis. J Ophthalmol. 2018;2018:2984934.

73. Vaphiades MS, Doyle JI. Peripheral cone dystrophy: a diagnostic improbability? J Neuroophthalmol. 2014;34(4):366-8.

74. Boon CJ, Klevering BJ, Leroy BP, et al. The spectrum of ocular phenotypes caused by mutations in the BEST1 gene. Prog Retin Eye Res. 2009;28(3): 187-205.

75. Laloum JE, Deutman AF. Peripheral vitelliform lesions in vitelliform macular dystrophy. J Fr Ophtalmol. 1991;14(2):74-8.

76. Shah D, Saurabh K, Roy R. Multimodal imaging in multifocal Best disease. Indian J Ophthalmol. 2018;66(9):1313-5.

77. Querques G, Regenbogen M, Soubrane G, et al. High-resolution spectral domain optical coherence tomography findings in multifocal vitelliform macular dystrophy. Surv Ophthalmol. 2009;54(2): 311-6. 